\title{
Goos-Hänchen Shifts in Graphene-Based Linear Barrier
}

\author{
Miloud Mekkaoui ${ }^{a}$, Radouane El Kinani $^{a}$ and Ahmed Jellal ${ }^{* a, b}$ \\ ${ }^{a}$ Laboratory of Theoretical Physics, Faculty of Sciences, Chouaib Doukkali University, \\ PO Box 20, 24000 El Jadida, Morocco \\ ${ }^{b}$ Saudi Center for Theoretical Physics, Dhahran, Saudi Arabia
}

\begin{abstract}
Using the energy spectrum of a system made of graphene subjected to a linear barrier potential, we study the Goos-Hänshen shifts. The calculation is done by first determining the corresponding phase shifts via the transmission and reflection probabilities. Numerical analysis shows that the Goos-Hänshen shifts depend strongly on the incident energy, barrier height and width, and vary positively or negatively under suitable conditions.
\end{abstract}

PACS numbers: 72.80.Vp, 73.21.-b, 71.10.Pm, 03.65.Pm

Keywords: graphene, linear potential, transmission, Goos-Hänchen shifts.

*a.jellal@ucd.ac.ma 


\section{Introduction}

Graphene is a honeycomb lattice in two dimensions, experimentally obtained in 2014 by Manchester group [1]. From time being graphene gives a good laboratory to test many theories like for instance quantum electrodynamics where Klein paradox takes place. This can experimentally be realized in graphene systems by considering Dirac fermions scattered by barrier potentials [2]. There is a big progress in studying quantum phenomena in graphene systems among them we cite the quantum version of the Goos-Hänchen (GH) effect originating from the reflection of particles from interfaces [3]. Many works in various graphene-based nanostructures, including single [4], double barrier [5], and superlattices [6], showed that the GH shifts can be enhanced by the transmission resonances and controlled by varying the electrostatic potential and induced gap [4]. Similar to those in semiconductors, the GH shifts in graphene can also be modulated by electric and magnetic barriers [7]. It has been reported that the GH shift plays an important role in the group velocity of quasiparticles along interfaces of graphene $\mathrm{p}-\mathrm{n}$ junctions $[8,9]$.

In our previous work [10], we have solved the 2D Dirac equation describing graphene in the presence of a linear vector potential. The discretization of the transverse momentum due to the infinite mass boundary condition reduced our 2D Dirac equation to an effective massive 1D Dirac equation with an effective mass equal to the quantized transverse momentum. We have used both a numerical Poincar map approach, based on space discretization of the original Dirac equation, and a direct analytical method. These two approaches have been used to study tunneling phenomena through a biased graphene strip. We have showed that the numerical results generated by the Poincar map are in complete agreement with the analytical results. In the second one [11], we have explored the zero, positive and negative quantum GH shifts of the transmitted Dirac carriers in graphene through a potential barrier with vertical magnetic field. Numerical results show that only one energy position at the zero GH shift exists and is highly dependent on the $y$-directional wave vector, the energy gap, the magnetic field and the potential. We have showed that the positive and negative GH shifts happen when the incident energy is more and less than the energy position at the zero GH shift, respectively. In addition, we found that there are two values of potential at the zero GH shifts, where a potential window can always keep the positive GH shifts.

Motivated by our previous work $[10,11]$, we consider Dirac fermions in graphene subjected to a linear barrier potential and study the GH shifts. From the solution of the energy spectrum we show how to derive the GH shifts as function of different physical parameters based on the phase shifts in transmission and reflection. To give a better understanding of our results, we give a numerical study based on different choices of the physical parameters. Among the obtained results we show that GH shifts can be controlled by linear barrier potential.

The present paper is organized as follows. In section 2, we set our problem and write down the corresponding Hamiltonian as well as the solutions of the energy spectrum for different regions composing our system. These will be used in section 3 to determine the transmission and reflection probabilities from which we derive the phase shifts. Using standard definition, we end up with the GH shifts in terms of the physical parameters characterizing our system. In section 4, we numerically analyze and discuss the phase shifts, GH shifts and transmission by considering suitable choices of the physical parameters. We conclude our results in the final section. 


\section{Energy spectrum of the system}

We consider massless Dirac fermions through a graphene scattered by linear barrier potential, as shown in Figure 1a, with incident energy $E$ and angle $\phi_{1}$ with respect to the $x$-direction, while they are free in the $y$-direction. Our system is made of three regions denoted by $j=1,2,3$ and each region is characterized by a given potential. The barrier regions are formally described by the Dirac-like Hamiltonian

$$
H=v_{F} \boldsymbol{\sigma} \cdot \mathbf{p}+V(x) \mathbb{I}_{2}
$$

where $v_{F} \approx 10^{6} \mathrm{~m} / \mathrm{s}$ is the Fermi velocity, $\boldsymbol{\sigma}=\left(\sigma_{x}, \sigma_{y}\right)$ are the Pauli matrices, $\mathbf{p}=-i \hbar\left(\partial_{x}, \partial_{y}\right), \mathbb{I}_{2}$ the $2 \times 2$ unit matrix, the electrostatic potential $V(x)=V_{j}$ in each scattering region is given by

$$
V(x)=V_{j}= \begin{cases}-\Lambda x+V_{0}, & 0 \leq x \leq d \\ 0, & \text { otherwise }\end{cases}
$$

with $\Lambda=\frac{V_{0}}{d}$, as presented schematically in Figure 1a. We illustrate the negative and positive GH shifts of Dirac fermions in transmission through the graphene linear barrier in Figure 1b.
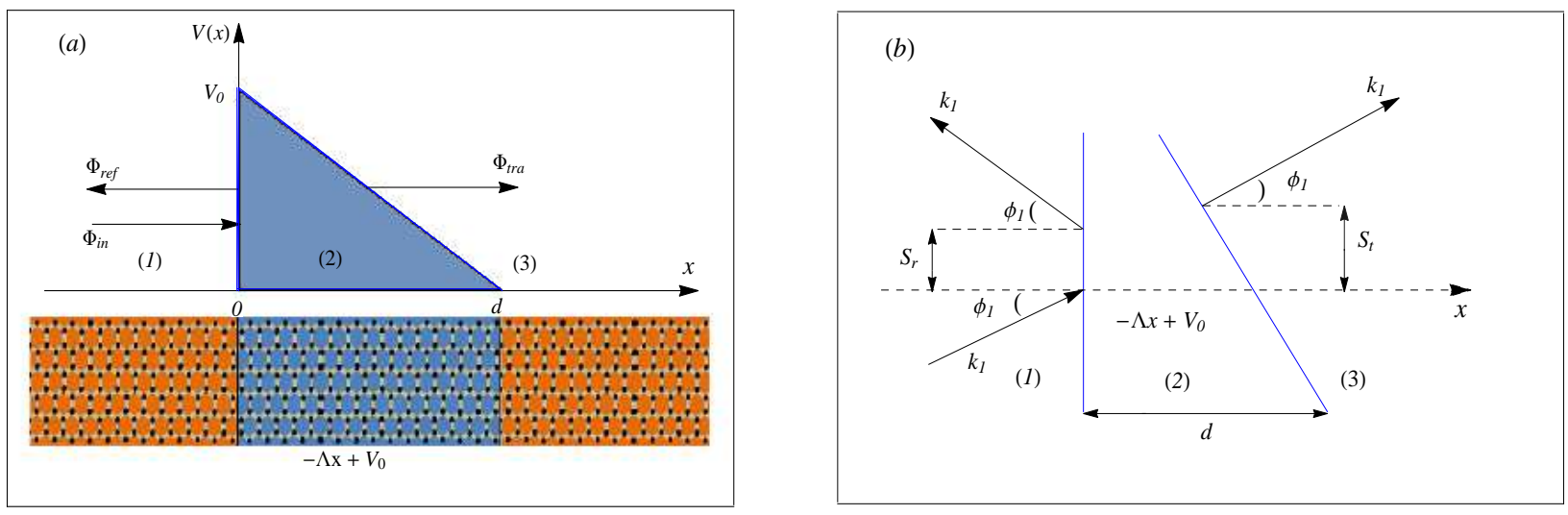

Figure 1: (a): Profile of a linear barrier potential of width $d$ and height $V_{0}$ applied to graphene composed of three regions. (b): The corresponding Goos-Hänchan shifts in transmission and reflection.

The time-independent Dirac equation for the spinor $\Phi(x, y)$ at energy $E=v_{F} \epsilon$ is given by

$$
\left[\boldsymbol{\sigma} \cdot \mathbf{p}+v_{j} \mathbb{I}_{2}\right] \Phi(x, y)=\epsilon \Phi(x, y)
$$

where we have rescaled the parameters $V_{j}=v_{F} v_{j}, \Lambda=v_{F} \varrho, \hbar=1, V_{0}=v_{F} v_{0}$ as well as the linear potential

$$
v_{j}= \begin{cases}-\varrho x+v_{0}, & 0 \leq x \leq d \\ 0, & \text { otherwise }\end{cases}
$$

Our system is supposed to have finite width $W$ with infinite mass boundary conditions on the wavefunction at the boundaries $y=0$ and $y=W$ along the $y$-direction $[12,13]$. This results in a quantization of the transverse momentum

$$
k_{y}=\frac{\pi}{W}\left(n+\frac{1}{2}\right), \quad n=0,1,2 \cdots
$$


Because of separability, we take the spinor $\Phi_{j}(x, y)=\left(\varphi_{j}^{+}(x), \varphi_{j}^{-}(x)\right)^{\dagger} e^{i k_{y} y}$ and solve the eigenvalue equation to obtain the upper and lower components of the eigenspinor in the incident and reflected region $1(x<0)$

$$
\begin{aligned}
& \Phi_{1}(x, y)=\left(\begin{array}{c}
1 \\
z_{1}
\end{array}\right) e^{i\left(k_{1} x+k_{y} y\right)}+r\left(\begin{array}{c}
1 \\
-z_{1}^{-1}
\end{array}\right) e^{i\left(-k_{1} x+k_{y} y\right)} \\
& z_{1}=s_{1} \frac{k_{1}+i k_{y}}{\sqrt{k_{1}^{2}+k_{y}^{2}}}
\end{aligned}
$$

where we have defined $s_{j}=\operatorname{sign}(E)$, and $r$ is a constant parameter while the corresponding dispersion relation can easily be obtained as

$$
\epsilon=s_{1} \sqrt{k_{1}^{2}+k_{y}^{2}}
$$

In region $2(0<x<d)$, we can express the general solution in terms of the parabolic cylinder function [10] and then the first component is

$$
\chi^{+}=c_{n 1} D_{\nu_{n}-1}(Q)+c_{n 2} D_{-\nu_{n}}\left(-Q^{*}\right)
$$

where $\nu_{n}=\frac{i k_{y}^{2}}{2 \varrho}, \epsilon_{0}=\epsilon-v_{0}$ and $Q(x)=\sqrt{\frac{2}{\varrho}} e^{i \pi / 4}\left(-\varrho x+\epsilon_{0}\right), c_{n 1}$ and $c_{n 2}$ are constants. We show that the second one takes the form

$$
\chi^{-}=-\frac{c_{n 2}}{k_{y}}\left[2\left(\epsilon_{0}-\varrho x\right) D_{-\nu}\left(-Q^{*}\right)+\sqrt{2 \varrho} e^{i \pi / 4} D_{-\nu_{n}+1}\left(-Q^{*}\right)\right]-\frac{c_{n 1}}{k_{y}} \sqrt{2 \varrho} e^{-i \pi / 4} D_{\nu_{n}-1}(Q) .
$$

The components of the spinor solution of the Dirac equation (3) in region 2 can be derived from (9) and (10) by setting

$$
\varphi^{+}(x)=\chi^{+}+i \chi^{-}, \quad \varphi^{-}(x)=\chi^{+}-i \chi^{-}
$$

which give the spinor

$$
\Phi_{2}(x, y)=a_{1}\left(\begin{array}{c}
\eta^{+}(x) \\
\eta^{-}(x)
\end{array}\right) e^{i k_{y} y}+a_{2}\left(\begin{array}{c}
\xi^{+}(x) \\
\xi^{-}(x)
\end{array}\right) e^{i k_{y} y}
$$

where the functions $\eta^{ \pm}(x)$ and $\xi^{ \pm}(x)$ read as

$$
\begin{aligned}
\eta^{ \pm}(x)= & D_{\nu_{n}-1}(Q) \mp \frac{1}{k_{y}} \sqrt{2 \varrho} e^{i \pi / 4} D_{\nu_{n}}(Q) \\
\xi^{ \pm}(x)= & \pm \frac{1}{k_{y}} \sqrt{2 \varrho} e^{-i \pi / 4} D_{-\nu_{n}+1}\left(-Q^{*}\right) \\
& \pm \frac{1}{k_{y}}\left(-2 i \epsilon_{0} \pm k_{y}+2 i \varrho x\right) D_{-\nu_{n}}\left(-Q^{*}\right)
\end{aligned}
$$

$a_{1}$ and $a_{2}$ are two constant parameters. Now solving the eigenvalue equation for the Hamiltonian (3) describing region $3(x>d)$, to end up with the spinor in the transmitted region

$$
\Phi_{3}(x, y)=t\left(\begin{array}{c}
1 \\
z_{1}
\end{array}\right) e^{i\left(k_{1} x+k_{y} y\right)}
$$

where $t$ is a constant parameter and $z_{1}$ is given by (7). 


\section{Goos-Hänshen shifts}

Before deriving the Goos-Hänchen $(\mathrm{GH})$ shifts in the beginning we determine the transmission and reflection probabilities for electrons in graphene subject to a linear barrier potential. For this, we write the obtained eigenspinors in matrix notation and impose the continuity of the wavefunctions at each interface of the linear barrier. We will use the above solutions to compute the corresponding transmission and reflection coefficient $(t, r)$ associated to phase shifts and build a bridge between quantum optics and Dirac fermions in graphene. The continuity of the spinor wavefunctions at each junction interface read as

$$
\Phi_{1}(0)=\Phi_{2}(0), \quad \Phi_{2}(d)=\Phi_{3}(d) .
$$

It is convenient to express these relationships in terms of $2 \times 2$ transfer matrices between different regions $M_{j j+1}$, which are

$$
\left(\begin{array}{c}
a_{j} \\
b_{j}
\end{array}\right)=M_{j, j+1}\left(\begin{array}{c}
a_{j+1} \\
b_{j+1}
\end{array}\right) .
$$

After some algebra, we obtain the total transfer matrix

$$
M=\prod_{j=1}^{4} M_{j j+1}
$$

as well as the relation between reflection and transmission amplitudes

$$
\left(\begin{array}{l}
1 \\
r
\end{array}\right)=M\left(\begin{array}{l}
t \\
0
\end{array}\right)
$$

where $M_{12}, M_{23}$ are transfer matrices that couple the wavefunction in the $j$-th region to the wavefunction in the $(j+1)$-th region. Explicitly, we have

$$
\begin{aligned}
M= & \left(\begin{array}{ll}
m_{11} & m_{12} \\
m_{21} & m_{22}
\end{array}\right) \\
M_{12} & =\left(\begin{array}{cc}
1 & 1 \\
z_{1} & -z_{1}^{*}
\end{array}\right)^{-1}\left(\begin{array}{cc}
\eta^{+}(0) & \xi^{+}(0) \\
\eta^{-}(0) & \xi^{-}(0)
\end{array}\right) \\
M_{23} & =\left(\begin{array}{ll}
\eta^{+}(d) & \xi^{+}(d) \\
\eta^{-}(d) & \xi^{-}(d)
\end{array}\right)^{-1}\left(\begin{array}{cc}
e^{i k_{1} d} & e^{-i k_{1} d} \\
z_{1} e^{i k_{1} d} & -z_{1}^{*} e^{-i k_{1} d}
\end{array}\right)
\end{aligned}
$$

with shorthand notations

$$
\eta^{ \pm}(0)=\eta_{0}^{ \pm}, \quad \eta^{ \pm}(d)=\eta_{d}^{ \pm}, \quad \xi^{ \pm}(0)=\xi_{0}^{ \pm}, \quad \xi^{ \pm}(d)=\xi_{d}^{ \pm} .
$$

Combining all to end up with the transmission and reflection amplitudes

$$
t=\frac{1}{m_{11}}, \quad r=\frac{m_{21}}{m_{11}}
$$

and after some lengthy algebra, we can show that they take the explicit forms

$$
\begin{aligned}
t & =\frac{e^{-i k_{1} d}\left[1+z_{1}^{2}\right]\left[\xi_{d}^{+} \eta_{d}^{-}-\xi_{d}^{-} \eta_{d}^{+}\right]}{\left[\xi_{0}^{+}+z_{1} \xi_{0}^{-}\right]\left[\eta_{d}^{-}-z_{1} \eta_{d}^{+}\right]-\left[\eta_{0}^{+}+z_{1} \eta_{0}^{-}\right]\left[\xi_{d}^{-}-z_{1} \xi_{d}^{+}\right]} \\
r & =\frac{\eta_{0}^{+} \xi_{d}^{-}+z_{1}\left(\eta_{d}^{-} \xi_{0}^{-}+\eta_{0}^{+} \xi_{d}^{+}-\eta_{d}^{+} \xi_{0}^{-}+\eta_{0}^{+} \xi_{d}^{-}\right)-z_{1}^{2}\left(\eta_{d}^{+} \xi_{0}^{+}-\eta_{0}^{+} \xi_{d}^{+}-\eta_{d}^{-} \xi_{0}^{+}\right)}{\left[\xi_{0}^{+}+z_{1} \xi_{0}^{-}\right]\left[\eta_{d}^{-}-z_{1} \eta_{d}^{+}\right]-\left[\eta_{0}^{+}+z_{1} \eta_{0}^{-}\right]\left[\xi_{d}^{-}-z_{1} \xi_{d}^{+}\right]}
\end{aligned}
$$


which be can written in complex notation as

$$
t=\rho_{t} e^{i \varphi_{t}}, \quad r=\rho_{r} e^{i \varphi_{r}}
$$

where the corresponding phase shifts are given by

$$
\varphi_{t}=\arctan \left(i \frac{t^{*}-t}{t+t^{*}}\right), \quad \varphi_{r}=\arctan \left(i \frac{\left(r^{*}-r\right)}{r+r^{*}}\right)
$$

as well as their modulus

$$
\rho_{t}=\sqrt{\Re^{2}[t]+\Im^{2}[t]}, \quad \rho_{r}=\sqrt{\left.\Re^{2}[r]\right)+\Im^{2}[r]} .
$$

Now from the above results, we can easily derive the corresponding transmission $T$ and reflection $R$ probabilities as

$$
T=\rho_{t}^{2}, \quad R=\rho_{r}^{2} .
$$

To study GH shifts in graphene scattered by linear barrier, we consider an incident, reflected and transmitted beams around some transverse wavevector $k_{y}=k_{y_{0}}$ and incident angle $\phi_{1}\left(k_{y_{0}}\right) \in\left[0, \frac{\pi}{2}\right]$, denoted by the subscript 0 . The incident one is given by

$$
\Psi_{i n}(x, y)=\int_{-\infty}^{+\infty} d k_{y} f\left(k_{y}-k_{y_{0}}\right) e^{i\left(k_{1}\left(k_{y}\right) x+k_{y} y\right)}\left(\begin{array}{c}
1 \\
e^{i \phi_{1}\left(k_{y}\right)}
\end{array}\right)
$$

and the reflected takes the form

$$
\Psi_{r e}(x, y)=\int_{-\infty}^{+\infty} d k_{y} r\left(k_{y}\right) f\left(k_{y}-k_{y_{0}}\right) e^{i\left(-k_{1}\left(k_{y}\right) x+k_{y} y\right)}\left(\begin{array}{c}
1 \\
-e^{-i \phi_{1}\left(k_{y}\right)}
\end{array}\right)
$$

where the reflection amplitude is $r\left(k_{y}\right)=|r| e^{i \varphi_{r}}$ (27). Here (31-32) are represented by writing the $x$-component of wavevector $k_{1}$ and $\phi_{1}$ in terms of the transverse wavevector $k_{y}$. The spinor plane waves are solutions of the eigenvalue equation (3) and $f\left(k_{y}-k_{y_{0}}\right)$ is the angular spectral distribution, assumed of Gaussian shape

$$
f\left(k_{y}-k_{y_{0}}\right)=w_{y} e^{-w_{y}^{2}\left(k_{y}-k_{y_{0}}\right)^{2}}
$$

with $w_{y}$ is the half beam width at waist [9]. We can approximate the $k_{y}$-dependent terms by a Taylor expansion around $k_{y}$ retaining only the first order term to get

$$
\begin{aligned}
& \phi_{1}\left(k_{y}\right) \approx \phi_{1}\left(k_{y_{0}}\right)+\left.\frac{\partial \phi_{1}}{\partial k_{y}}\right|_{k_{y_{0}}}\left(k_{y}-k_{y_{0}}\right) \\
& k_{1}\left(k_{y}\right) \approx k_{1}\left(k_{y_{0}}\right)+\left.\frac{\partial k_{1}}{\partial k_{y}}\right|_{k_{y_{0}}}\left(k_{y}-k_{y_{0}}\right) .
\end{aligned}
$$

In the same way, we write the transmitted beam as

$$
\Psi_{t r}(x, y)=\int_{-\infty}^{+\infty} d k_{y} t\left(k_{y}\right) f\left(k_{y}-k_{y_{0}}\right) e^{i\left(k_{1}\left(k_{y}\right) x+k_{y} y\right)}\left(\begin{array}{c}
1 \\
e^{i \phi_{1}\left(k_{y}\right)}
\end{array}\right)
$$

where the transmitted coefficient $t\left(k_{y}\right)=|t| e^{i \varphi_{t}}$ (27) will be calculated through the use of boundary conditions. The stationary-phase approximation indicate that the GH shifts are equal to the negative gradients of transmission and reflection phases with respect to $k_{y}$. They are given by

$$
S_{t}=-\left.\frac{\partial \varphi_{t}}{\partial k_{y}}\right|_{k_{y 0}}, \quad S_{r}=-\left.\frac{\partial \varphi_{r}}{\partial k_{y}}\right|_{k_{y 0}} .
$$


Next we will numerically analyze and discuss the GH shifts for Dirac fermions in graphene scattered by a linear barrier. This will be done by tuning on different physical parameters characterizing our system under suitable conditions.

\section{Results and discussions}

Figure 2(a) shows the phase shifts $\varphi_{t}$ of the transmitted beam versus energy potential $v_{0}$ for a linear barrier with incident energy $\epsilon=20, k_{y}=1$ and two different values of distance $d=5$ (red line), $d=10$ (green line). They are oscillating periodically from positive to negative values with the same amplitudes and change the phase as long as distance $d$ increases. Figure 2(b) presents transmission and reflection probabilities as function of energy potential $v_{0}$ with the same conditions as before. We observe that $T$ takes maximum value for $v_{0}$ far away from incident energy $\epsilon$. It then decreases sharply for $v_{0}>\epsilon-2 k_{y}$ until it reaches a relative minimum and then begins to increase in an oscillatory manner. The inverse behavior of $T$ is exhibited by the reflection $R$ showing that the conservation $T+R=1$ is well satisfied.
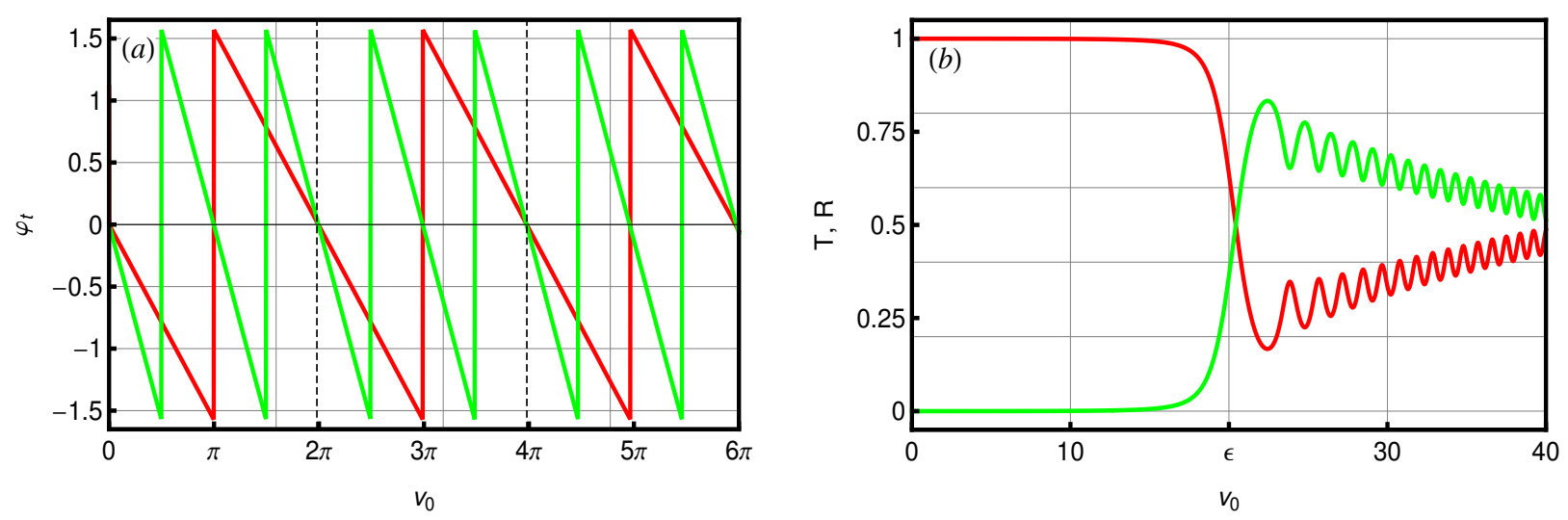

Figure 2: (Color online) (a): Phase shifts $\varphi_{t}$ of the transmitted beam versus energy potential $v_{0}$ graphene linear barrier with $k_{y}=1, \epsilon=20, d=5$ (red line), $d=10$ (green line). (b): Transmission (red line) and reflection (green line) probabilities versus $v_{0}$ with $d=10, k_{y}=1, \epsilon=20$.

Figure 3 presents the GH shifts $\left\{S_{t}, S_{r}\right\}$ and probability $\{T, R\}$ as function of energy potential $v_{0}$, with the parameters $k_{y}=1, \epsilon=20, d=0.2$ (magenta line), $d=2$ (blue line), $d=6$ (green line), $d=10$ (red line). From Figure 3(a), we observe that $S_{t}$ increase to reach a peak as long as $v_{0}$ increases and then rapidly decrease to zero after that it oscillate by decreasing in negative regime. In other words, the GH shifts can be changed from positive to negative by controlling the strength of the linear potential. However, the GH shifts finally become positive with increasing the strength of the energy potential $v_{0}$ up to $v_{0}>\epsilon$ and after decrease in an oscillatory manner toward negative regime. From Figure 3(b) one sees that $S_{r}$ at $v_{0}<\epsilon$ firstly become negative and null asymptotically, after increase up to $\epsilon=v_{0}$. However in the opposite case, namely $v_{0}>\epsilon, S_{r}$ decrease and finally become negative then begin to increase in an oscillatory manner. We clearly see that the GH shifts behave according to the variation of the corresponding transmission and reflections probabilities as shown in Figures $3 \mathrm{c}$ and $3 \mathrm{~d}$, respectively. 

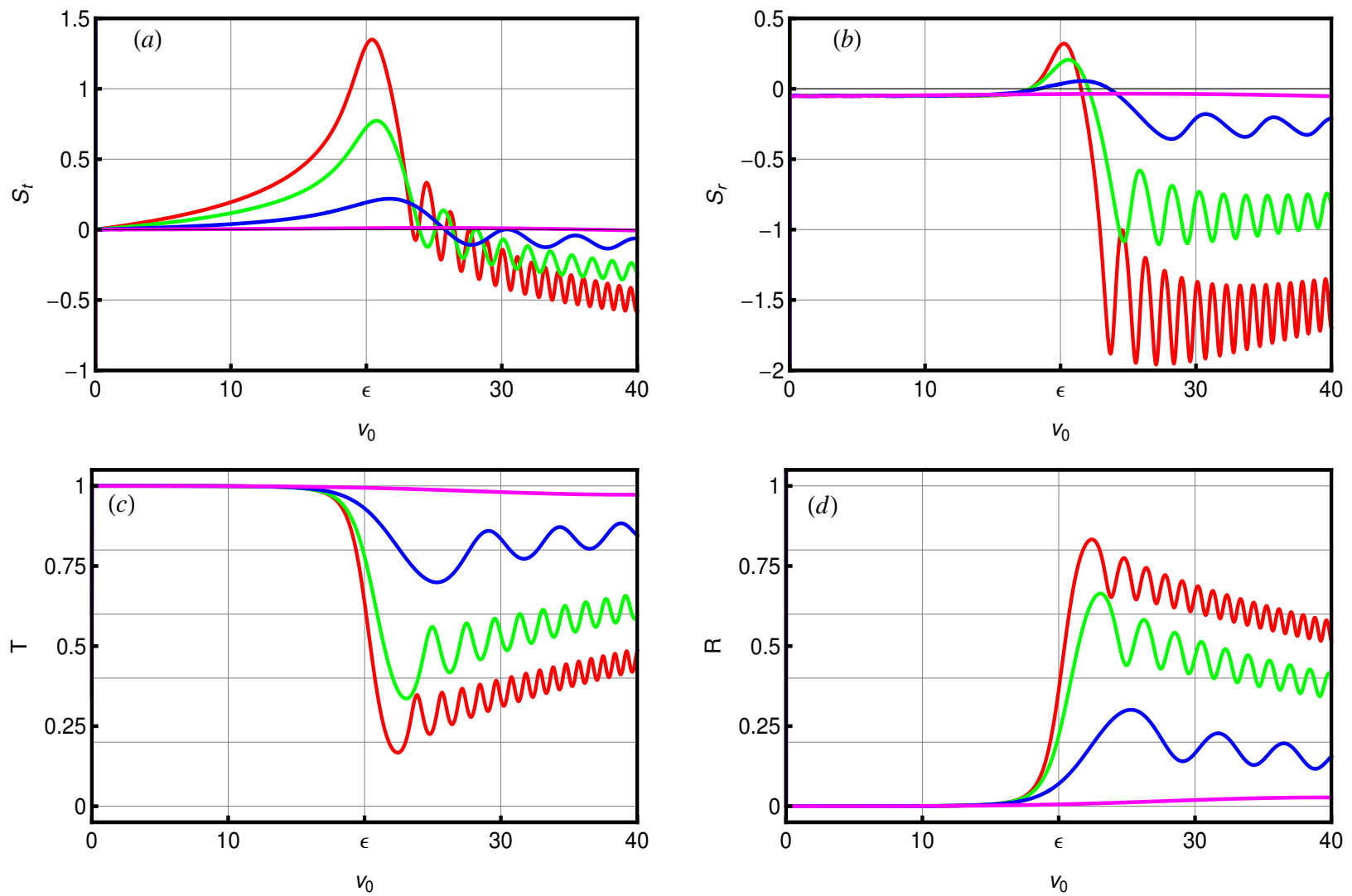

Figure 3: (color online) (a): GH shifts $S_{t}$ in transmission and transmission probability $T$, (b): GH shifts $S_{r}$ in reflection and reflection probability $R$ versus energy potential $v_{0}$ with $k_{y}=1, \epsilon=20, d=0.2$ (magenta line), $d=2$ (blue line), $d=6$ (green line), $d=10$ (red line).

In Figure 4 we present the GH shifts $\left\{S_{t}, S_{r}\right\}$ and probability $\{T, R\}$ as function of energy $\epsilon$, with $k_{y}=1, v_{0}=20, d=7$ (green line), $d=10$ (red line). It is clearly seen that at the Dirac points $\epsilon=v_{0}$, the GHL shifts change their signs. This change shows clearly that they are strongly dependent on the barrier heights. In Figure 4(a), we observe that the negative GH shifts $S_{t}$ in transmission at $\epsilon<v_{0}$ are negatively enhanced, firstly increase accompanied with slight oscillations and finally become positive with the strength of linear barrier. However, the positive GH shifts in transmission at $\epsilon>v_{0}$ decrease, to reach asymptotically a null value. We observe that for a given energy, $S_{t}$ in transmission decrease if $d$ decreases and then vanishes. Note that below a certain critical energy $\epsilon=k_{y}$ the transmission is almost zero, then it starts oscillating with frequency increases with increase of distance $d$, which is the size of the region subject to the electric field. Also the transmission increases with $d$ decreases in the range $k_{y}<\epsilon<v_{0}+2 k_{y}$ and reaches unity for energies above $\epsilon>v_{0}+2 k_{y}$. Figure 4(b) shows the GH shifts $S_{r}$ in reflection and reflection probability $R$ as function of incident energy $\epsilon$. The negative and positive GH shifts at $\epsilon<v_{0}$ are negatively enhanced and then begin to increase in an oscillatory manner. However, the positive GH shifts at $\epsilon>v_{0}$ firstly decrease and finally become negative and null asymptotically. We observe that the reflection $R$ decreases a long as $d$ decreases and reaches zero value for energies above $\epsilon>v_{0}+2 k_{y}$. We see that the GH shifts can be enhanced by adjusting the incident energy or tuning on the distance of region where the barrier is applied. This show that the GH shifts can be controlled by linear potential. 

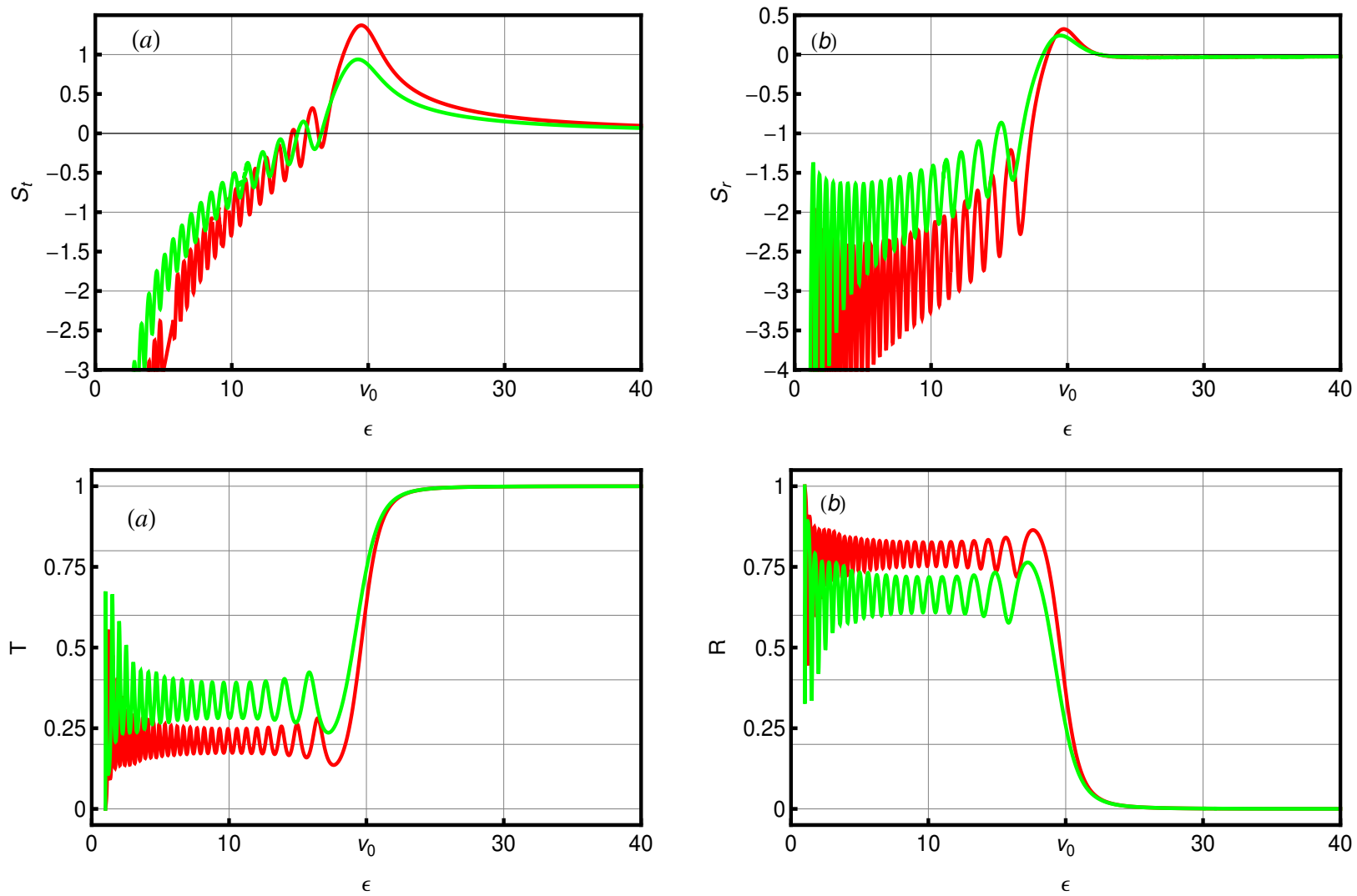

Figure 4: (color online) (a): GH shifts $S_{t}$ in transmission and transmission probability $T$, (b): GH shifts $S_{r}$ in reflection and reflection probability $R$ versus incident energy $\epsilon$ with $k_{y}=1, v_{0}=20, d=7$ (green line), $d=10$ (red line).
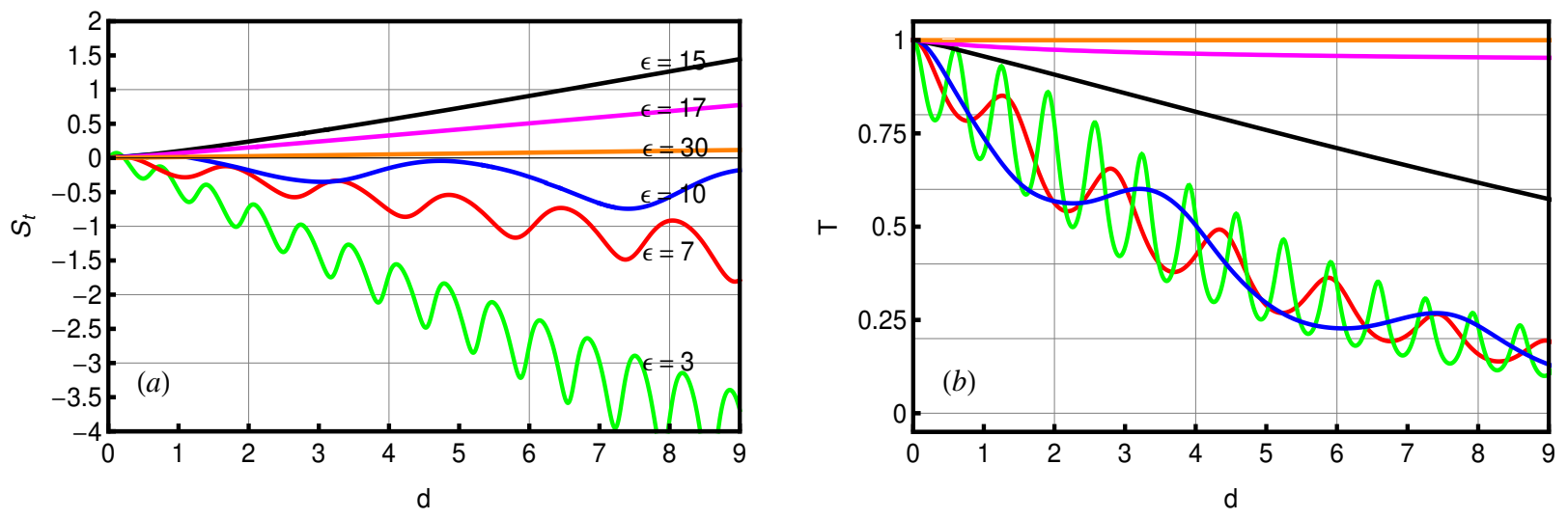

Figure 5: (color online) (a/b): GH shifts in transmission and transmission coefficient $S_{t} / T$ versus barrier width $d$, with $k_{y}=1, v_{0}=15$ and six values of incident energy $\epsilon=\{3,7,10,15,17,30\}$.

Figure 5 shows the GH shifts $S_{t}$ in transmission and the corresponding transmission $T$ as function of the barrier width $d$ with $k_{y}=1, v_{0}=15$ and different values of incident energy $\epsilon=\{3,7,10,15,17,30\}$. In Figure 5(a), the GH shifts increase as long as $\epsilon$ increases in the range $\epsilon<v_{0}$ with slight oscillations and finally become negative. However, in the range $\epsilon \geq v_{0}$ the positive GH shifts decrease once $\epsilon$ increases and there is no oscillation. Figure 5(b) shows transmission oscillating for $\epsilon<v_{0}$, which 
increases with $\epsilon$ and is total in the case $\epsilon \geq v_{0}$.

Figure 6 presents the transmission, GH shifts and phase shifts as a function of the incident angle $\phi_{1}$ for $\epsilon=2, v_{0}=5$ and different values of $d=\{3,6,10\}$. From Figure 6(a), we see that the perfect transmission occurs at different angles and vice verse. It is observed that, the transmission is always total for a normal incidence angle and vanishes for specific values, it decreases by increasing the value of barrier width $d$. We observe that the curve of $T$ is symmetric with respect to the normal incidence around the Dirac point. From Figure 6(b) one sees that the GH shifts can be changed from negative to positive according to variation of $\phi_{1}$. Indeed, the GH shifts are positive as long as the condition $\phi_{1}<0$ is satisfied and negative otherwise, namely $\phi_{1}>0$. The GH shifts are also symmetric with respect to the normal incidence around the Dirac point. In Figure 6(c), we observe that phase shifts are oscillating according the value taken by transmission and show symmetric behavior. All Figures show a symmetry at normal incidence angle $\phi_{1}=0$ separating positive and negative behavior of the GH shifts in transmission.
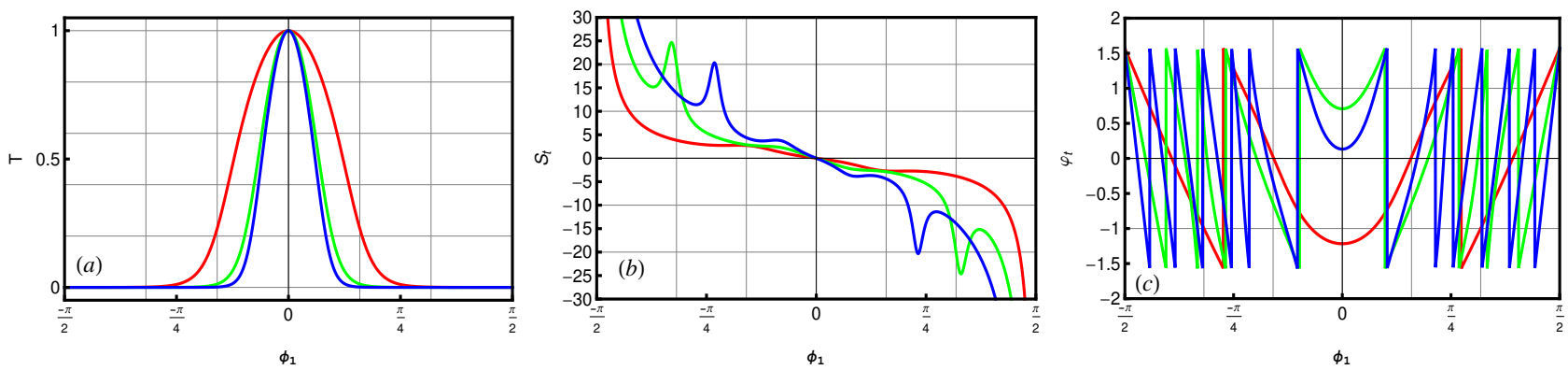

Figure 6: (color online) (a): Transmission probability $T\left(\phi_{1}\right),(\mathrm{b})$ : GH shifts in transmission $S_{t}\left(\phi_{1}\right),(\mathrm{c})$ : phase shifts $\varphi_{t}\left(\phi_{1}\right)$ versus incidence angle $\phi_{1}$ with $v_{0}=5, \epsilon=2, d=3$ (red line), $d=6$ (green line), $d=10$ (blue line).

\section{Conclusion}

We have studied the Goos-Hänchen shifts for Dirac fermions in graphene scattered by a linear barrier potential along the $x$-direction and free in the $y$-direction. After setting the solutions of the energy spectrum for three regions composing our system, we have calculated the phase shifts of the transmitted and reflected beams via the transmission and reflection coefficients. These phases have been used to obtain the Goos-Hänchen shifts in transmission and reflection as function of a set of physical parameters characterizing our system.

Subsequently, we have presented different numerical results to underline the basic features of our system. Indeed, the phase shifts of transmitted beam showed oscillations with the same amplitude, as long as the barrier width increases the phase shifts behave in the same way but with some phases. Later on we have analyzed the GH shifts in terms of incident energy, barrier width and energy potential. We have observed that $\mathrm{GH}$ shifts are balancing from positive to negative regimes by exhibiting different behaviors. We hope that our graphene-like results could present a possible way to simulate the quantum transport and GH shifts of Dirac fermions in graphene through a linear barrier potential by employing the optical technology. 


\section{Acknowledgment}

The generous support provided by the Saudi Center for Theoretical Physics (SCTP) is highly appreciated by all authors.

\section{References}

[1] K. S. Novoselov, A. K. Geim, S. V. Morozov, D. Jiang, Y. Zhang, S. V. Dubonos, I. V. Grigorieva and A. A. Firsov, Science 306, 666 (2004).

[2] N. Stander, B. Huard and D. Goldhaber-Gordon, Phys. Rev. Lett. 102, 026807 (2009).

[3] F. Goos and H. Hänchen, Ann. Phys. 436, 333 (1947).

[4] X. Chen, J.-W. Tao, and Y. Ban, Eur. Phys. J. B 79, 203 (2011).

[5] Y. Song, H-C. Wu and Y. Guo, Appl. Phys. Lett. 100, 253116 (2012).

[6] X. Chen, P-L. Zhao, X-J. Lu and L-G. Wang, Eur. Phys. J. B 86, 223 (2013).

[7] M. Sharma and S. J. Ghosh, J. Phys.: Condens. Matter 23, 055501 (2011).

[8] L. Zhao and S. F. Yelin, Phys. Rev. B 81, 115441 (2010).

[9] C. W. J. Beenakker, R. A. Sepkhanov, A. R. Akhmerov and J. Tworzydlo, Phys. Rev. Lett. 102, 146804 (2009).

[10] H. Bahlouli, E. B. Choubabi, A. El Mouhafid and A. Jellal, Solid State Communications 151, (2011) 1309.

[11] A. Jellal, Y. Wang, Y. Zahidi and M. Mekkaoui, Physica E 68, 53 (2015) 53.

[12] M. V. Berry and R. J. Modragon, Proc. R. Soc. London Ser. A 412, 53 (1987).

[13] J. Tworzydlo, B. Trauzettel, M. Titov, A. Rycerz and C. W. J. Beenakker, Phys. Rev. Lett. 96, $246802(2006)$. 